\title{
PHASE TRANSITIONS IN MAGNETIC AND SUPERCONDUCTING SYSTEMS WITH FLUCTUATING VALENCE. CHEMICAL POTENTIAL EVIDENCE
}

\author{
E. GoseaWsKa ${ }^{a}$, M. MATLAK ${ }^{a *}$ AND Kh. Eid ${ }^{b}$ \\ ${ }^{a}$ Institute of Physics, Silesian University \\ Uniwersytecka 4, 40-007 Katowice, Poland \\ ${ }^{b}$ Physics Department, Faculty of Education \\ Ain-Shams University, Roxy, Cairo, Egypt
}

(Received November 18, 1999)

\begin{abstract}
We investigate the critical behaviour of the chemical potential and average occupation numbers using the extended $s-f$ model with intersite Cooper pairing for systems with fluctuating valence. The model is able to describe phase transitions from normal ferromagnet to normal paramagnet at $T=T_{\mathrm{C}}$, from superconducting paramagnet to normal paramagnet at $T=T_{\mathrm{S}}$, as well as reentrant phase transitions with three critical temperatures $T_{\mathrm{S}_{1}}, T_{\mathrm{C}}$ and $T_{\mathrm{S}_{2}}\left(T_{\mathrm{S}_{1}}<T_{\mathrm{C}}<T_{\mathrm{S}_{2}}\right)$. Present investigation, as well as recent results obtained for another models suggest one-to-one correspondence between critical temperatures of the system and kinks appearing in the temperature dependence of the chemical potential and average occupation numbers. This, in turn, indicates a possibility to apply the measurement of the chemical potential vs. temperature as an experimental universal tool when looking for phase transitions in solids.
\end{abstract}

PACS numbers: $75.30 . \mathrm{Kz}, 75.40 . \mathrm{Cx}$

\section{Introduction}

Recent theoretical investigations [1-5] showed that the temperature dependence of the average occupation numbers and of the chemical potential can be used to detect phase transitions (temperature and concentration driven, structural phase transitions included). The mentioned quantities, exhibiting kinks in the critical points, clearly indicate critical temperatures (or concentrations) of the considered systems. From the theoretical point of view there is no possibility to

*e-mail: matlak@server.phys.us.edu.pl 
give a general proof of this fact. We can, however, study many different, microscopical models looking for this general tendency. Such investigations seem to be very important at present because till now [1-5] only model systems with integer valence have been studied. Therefore, it is necessary to see whether the mentioned critical behaviour of the average occupation numbers (critical electron redistribution effect) and of the chemical potential also holds for systems with intermediate valence. In Ref. [6] the extended $s-f$ model with intersite Cooper pairing has been applied to study the competition between ferromagnetism and superconductivity for system exhibiting fluctuating valence. Using this model in the present paper we show that the effect of kinks at critical points is also present in the case of such systems for all phase transitions (normal ferromagnet-normal paramagnet, superconducting paramagnet-normal paramagnet and reentrant phase transitions) possible to appear within the model.

\section{Model, calculations and conclusions}

The Hamiltonian of the extended $s-f$ model with intersite Cooper pairing (see Ref. [6]) can be presented in the following form (grand canonical ensemble):

$$
H=H_{f}+H_{d}+H_{f-d}+H_{N}
$$

where

$$
\begin{aligned}
& H_{f}=E_{f} \sum_{i, \sigma} n_{i, \sigma}^{f}+U \sum_{i} n_{i, \uparrow}^{f} n_{i, \downarrow}^{f}+\sum_{i, j} \Delta_{i, j}^{(f)} f_{i, \uparrow}^{+} f_{j, \downarrow}^{+}+\sum_{i, j} \Delta_{i, j}^{*(f)} f_{j, \downarrow} f_{i, \uparrow}, \\
& H_{d}=\sum_{i, j, \sigma} t_{i, j}^{d} d_{i, \sigma}^{+} d_{j, \sigma}+\sum_{i, j} \Delta_{i, j}^{(d)} d_{i, \uparrow}^{+} d_{j, \downarrow}^{+} \sum_{i, j} \Delta_{i, j}^{*(d)} d_{j, \downarrow} d_{i, \uparrow}, \\
& H_{f-d}=-\frac{g}{2} \sum_{i}\left[f_{i, \uparrow}^{+} f_{i, \downarrow} d_{i, \downarrow}^{+} d_{i, \uparrow}+f_{i, \downarrow}^{+} f_{i, \uparrow} d_{i, \uparrow}^{+} d_{i, \downarrow}+\frac{1}{2}\left(n_{i, \uparrow}^{f}-n_{i, \downarrow}^{f}\right)\left(n_{i, \uparrow}^{d}-n_{i, \downarrow}^{d}\right)\right] \\
& \quad+V \sum_{i}\left(f_{i, \sigma}^{+} d_{i, \sigma}+d_{i, \sigma}^{+} f_{i, \sigma}\right), \\
& H_{N}=-\mu \sum_{i, \sigma}\left(n_{i, \sigma}^{f}+n_{i, \sigma}^{d}\right),
\end{aligned}
$$

where according to [7]

$$
\begin{aligned}
& \Delta_{i, j}^{(f)}=J_{i, j}\left\langle f_{j, \downarrow} f_{i, \uparrow}\right\rangle, \\
& \Delta_{i, j}^{(d)}=R_{i, j}\left\langle d_{j, \downarrow} d_{i, \uparrow}\right\rangle .
\end{aligned}
$$

The Hamiltonian (2) describes the subsystem of $4 f(5 f)$ electrons and contains the following model parameters: $E_{f}$ (position of the $4 f(5 f)$ level), $U$ (Coulomb repulsion) and $J_{i, j}$ (intersite Cooper pairing for $4 f(5 f)$ electrons (cf. Ref. [7])). The subsystem of conduction electrons describes the Hamiltonian (3) with model parameters: $t_{i, j}^{d}$ (hopping integral for conduction electrons: we apply the energy scale where $t_{i, i}^{d}=0$ ) and $R_{i, j}$ (intersite Cooper pairing for conduction electrons, similar to $J_{i, j}$ ). The Hamiltonian (4) describes the mutual interaction 
between $4 f(5 f)$ electrons and conduction electrons with model parameters: $g(s-f$ coupling constant (local exchange integral)) and $V$ (intrasite hybridization parameter). $\mu$ in Eq. (5) denotes the chemical potential. The indices $i$ and $j$ number the lattice sites. The construction operators are denoted by $f_{i, \sigma}\left(f_{i, \sigma}^{+}\right)$and $d_{i, \sigma}\left(d_{i, \sigma}^{+}\right)$ for $4 f(5 f)$ electrons and conduction electrons $(\sigma=\uparrow, \downarrow)$, respectively. From the beginning we have assumed the intersite Cooper pairing between $4 f(5 f)$ electrons and conduction electrons (see (2) and (3)) because assuming the Cooper pairing for conduction electrons only (as usually) due to the hybridization term (see (4)) the superconductivity will automatically appear also in the $4 f(5 f)$ subsystem and vice versa. The model (1) without third and fourth term in (2) and second and third term in (3) has been used to describe the properties of lanthanide or actinide based materials with fluctuating valence (cf. Refs. [8-11]). Including the intersite Cooper pairing terms between $4 f(5 f)$ and conduction electrons in (2) and (3) we enhance the applicability of the model (1) to magnetic and superconducting systems (see Refs. [6], [12] and [13]) with fluctuating valence.

To calculate the chemical potential we use the constraint

$$
\sum_{\sigma}\left(\left\langle n_{\sigma}^{f}\right\rangle+\left\langle n_{\sigma}^{d}\right\rangle\right)=n
$$

where $\left\langle n_{\sigma}^{f, d}\right\rangle$ are average occupation numbers for both $4 f(5 f)$ and conduction electrons $(\sigma=\uparrow, \downarrow)$ and $n$ is the average number of electrons per lattice site. Applying the same approximation as in Ref. [6] and standard Green's function technique ${ }^{\dagger}[14]$, we can finally find the system of nine implicit equations describing the thermodynamics of the model system (1). In Fig. 1 we present the calculated Curie temperature $T_{\mathrm{C}}$ and upper superconducting transition temperature $T_{\mathrm{S}_{2}}$ as function of the $4 f(5 f)$ level position $E_{f}\left(J_{i, j} \neq 0, R_{i, j} \neq 0\right)$ for given value of $n=0.6$. The lower superconducting transition temperature $T_{\mathrm{S}_{1}}$ lying a little below $T_{\mathrm{C}}$-curve is not marked in Fig. $1\left(T_{\mathrm{S}_{1}} \approx T_{\mathrm{C}}\right.$ in the scale of Fig. 1 but in reality $\left.T_{\mathrm{S}_{1}}<T_{\mathrm{C}}\right)$. Figure 1 presents at the same time the phase diagram of the system. Our special interest is to investigate the temperature behaviour of the average occupation numbers

$$
n_{f, d}=\sum_{\sigma}\left\langle n_{\sigma}^{f, d}\right\rangle
$$

and the chemical potential $\mu$. This behaviour can be demonstrated in three limiting cases:

(i) phase transition: normal ferromagnet - normal paramagnet (we exclude intersite Cooper pairing $\left(J_{i, j}=R_{i, j}=0\right)$ ),

(ii) phase transition: superconducting paramagnet - normal paramagnet (we use the cross-section $E_{f}=-0.75 \mathrm{eV}$ from Fig. 1),

(iii) reentrant phase transitions with three critical temperatures $T_{\mathrm{S}_{1}}, T_{\mathrm{C}}$ and $T_{\mathrm{S}_{2}}$ (we use the cross-section $E_{f}=-1 \mathrm{eV}$ from Fig. 1).

The calculated temperature dependence of the average occupation numbers (9) and of the chemical potential $\mu$ for three limiting cases (i), (ii), and (iii)

\footnotetext{
tFor the model Hamiltonian (1) it is possible to derive an exact equation connecting the Green functions belonging to both subsystems. The approximate Green functions, calculated in this paper exactly fulfil this exact equation.
} 


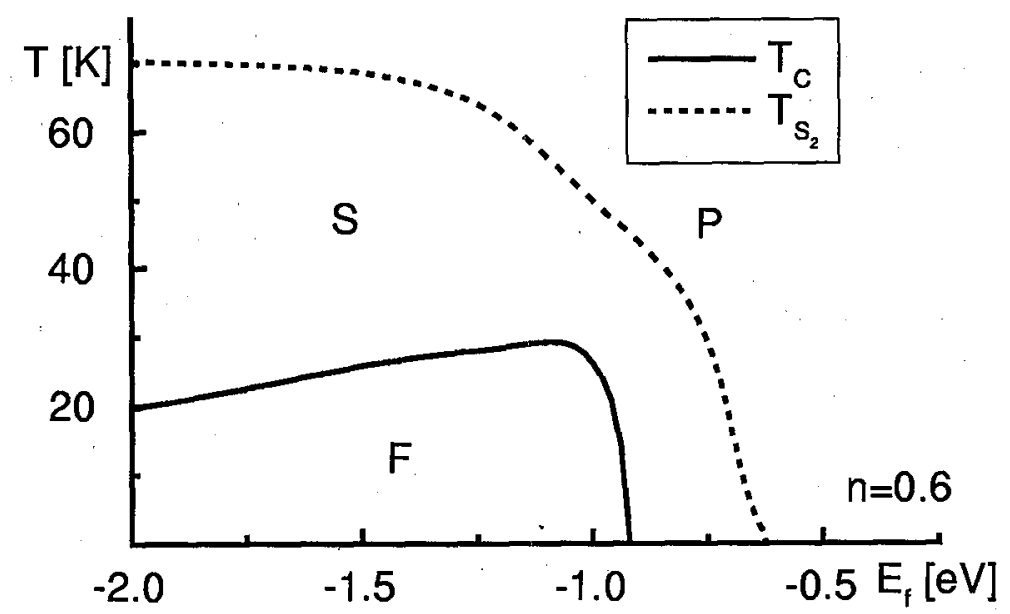

Fig. 1. Phase diagram of the model in the $\left(T, E_{f}\right)$ plane for $n=0.6$. Model parameters: $W=2 \mathrm{eV}$ (band width of the conduction band), $V=0.1 \mathrm{eV}, g=0.2 \mathrm{eV}$, $U=10 \mathrm{eV}, J_{0}\left(=\sum_{j[\mathrm{n} . \mathrm{n} .]} J_{i, j}\right)=R_{0}\left(=\sum_{j[\mathrm{n} . \mathrm{n} .]} R_{i, j}\right)=0.15 \mathrm{eV} . \mathrm{F}$ - ferromagnetic $\mathrm{S}$ - superconducting and $\mathrm{P}$ - paramagnetic phases.

is depicted in Figs. 2a-c, respectively. All critical temperatures: $T_{\mathrm{C}}$ (Fig. 2a), $T_{\mathrm{S}}$ (Fig. 2b), and $T_{\mathrm{S}_{1}}, T_{\mathrm{C}}, T_{\mathrm{S}_{2}}$ (Fig. 2c) can easily be identified from the plots, because at each critical temperature the average occupation numbers (9) and the chemical potential $\mu$ change their slopes (small but visible kinks). The phase transitions take place at such a critical temperature for which average occupation numbers (critical electron redistribution within the constraint (8)) and the chemical potential $\mu$ reach their critical values. The changes in the slopes at all critical points can even better be displayed when instead to plot the mentioned quantities as functions of temperature we plot their temperature derivatives (not depicted here). In this latter case in all critical points we should see the jumps of the corresponding temperature derivatives (in the case of the constraint (8) $\partial n_{f} / \partial T=-\partial n_{d} / \partial T$ at each critical point). Looking at the curves in Figs. 2a-c we can see that the effect of kinks, present for systems with integer valence [1-5] takes also place for systems with fluctuating valence. In other words, the effect seems to be generally present, and, when it appears in our model calculation, we expect that the effect of kinks should also be seen in experiments.

Especially interesting and useful from the experimental point of view should be the measurement of the temperature dependence of the chemical potential (or its temperature derivative (more sensitive method)) because according to our calculations the kinks (or jumps in $\partial \mu / \partial T$ ) clearly indicate critical temperatures. The measurement of the chemical potential vs. temperature can be performed with the use of the photoelectron spectroscopic methods, presented in Ref. [15]. To show that very small changes in the slope of the chemical potential can be experimentally measured with sufficient accuracy let us recall the results obtained for high temperature superconductor $\mathrm{YBa}_{2} \mathrm{Cu}_{3} \mathrm{O}_{7-\delta}$. In Refs. $[16,17]$ the authors have predicted 

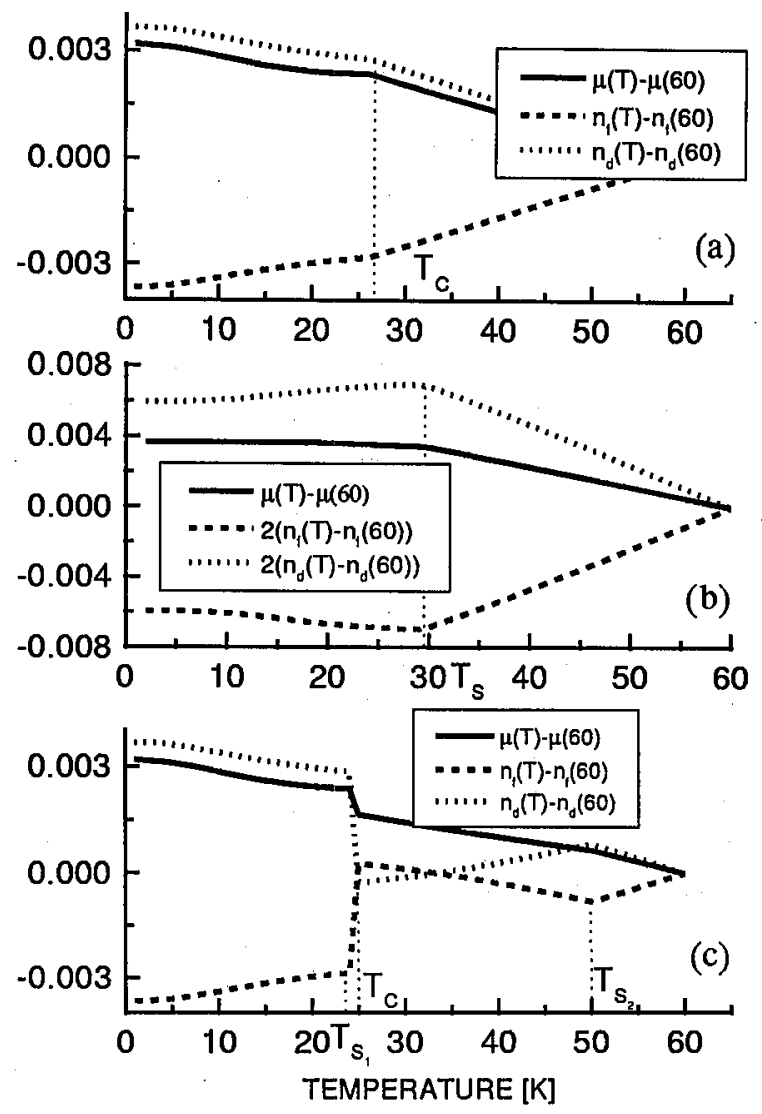

Fig. 2. Plot of the average occupation numbers (9) and of the chemical potential $\mu[\mathrm{eV}]$ vs. temperature for three limiting cases (i), (ii), and (iii) in (a), (b), (c) respectively. Model parameters for (a): $E_{f}=-1 \mathrm{eV}, J_{0}=R_{0}=0$. Parts (b) and (c) display the cross-sections $E_{f}=-0.75 \mathrm{eV}$ and $E_{f}=-1 \mathrm{eV}$ from Fig. 1, respectively. The other model parameters as in Fig. 1.

the change in the slope of the chemical potential at the critical temperature $T_{\mathrm{S}}$. With the use of the phenomenological Ginzburg-Landau theory [18] the authors of Ref. [19] could even connect the jump in $\partial \mu / \partial T$ at $T_{\mathrm{S}}$ with the jump in the specific heat. The straightforward measurement of the chemical potential vs. temperature, performed for high temperature superconductor $\mathrm{YBa}_{2} \mathrm{Cu}_{3} \mathrm{O}_{7-\delta}\left(T_{\mathrm{S}} \approx 90 \mathrm{~K}\right)$ clearly showed a kink at $T_{\mathrm{S}}$ (see Figs. 1, 2 in Ref. [20]) in agreement with Ref. [16]. This example in connection with the results of Refs. [1-5] and the present paper (fluctuating valence systems) suggests an experimental possibility to apply the measurements of the chemical potential vs. temperature (concentration, pressure, external fields etc.) for all types of phase transitions (temperature or concentration driven, external field induced transitions etc.) for magnetic, superconducting, ferroelectric systems (structural phase transitions included) as a universal tool to detect all critical temperatures (concentrations, critical fields, etc.) characterizing real materials. 


\section{References}

[1] M. Matlak, M. Pietruszka, Physica C 311, 151 (1999).

[2] M. Matlak, M. Pietruszka, J. Alloys Comp. 291, 21 (1999).

[3] M. Matlak, E. Gosławska, B. Grabiec, Kh. Eid, to be published in Acta Phys. Pol. A.

[4] M. Matlak, M. Pietruszka, Acta Phys. Pol. A 97, 253 (2000).

[5] E. Gosławska, M. Matlak, Phys. Status Solidi $B$, in press.

[6] E. Gosławska, M. Matlak; J. Magn. Magn. Mater. 187, 51 (1998).

[7] R. Micnas, J. Ranninger, S. Robaszkiewicz, Rev. Mod. Phys. 62, 113 (1990).

[8] M. Matlak, W. Nolting, Z. Phys. B 55, 103 (1984).

[9] M. Matlak, W. Nolting, J. Magn. Magn. Mater. 60, 285 (1986).

[10] M. Matlak, Solid State Commun. 65, 1575 (1988).

[11] M. Matlak, M. Pietruszka, J. Magn. Magn. Mater. 153, 115, 347 (1996).

[12] M.B. Maple, Physica $C$ 215, 110 (1995).

[13] J.A. Sauls, Adv. Phys. 43, 113 (1994).

[14] A.A. Abrikosov, L.P. Gorkov, I.E. Dzyaloshinskii, Quantum Field Theoretical Methods in Statistical Physics, Pergamon Press, Oxford 1965.

[15] S. Hüfner, Photoelectron Spectroscopy, Springer-Verlag, Berlin 1996.

[16] S. Robaszkiewicz, R. Micnas, K.A. Chao, Phys. Rev. B 26, 3915 (1982).

[17] J. Czerwonko, Acta Phys. Pol. B 29, 3885 (1998).

[18] W.L. Ginzburg, L.D. Landau, Sov. Phys.-JETP 20, 1064 (1950).

[19] D. van der Marel, G. Ritveld, Phys. Rev. Lett. 69, 2575 (1992).

[20] G. Rietveld, N.Y. Chen, D. van der Marel, Phys. Rev. Lett. 69, 2578 (1992). 